\title{
Real-Time Prediction of Brain Shift Using Nonlinear Finite Element Algorithms
}

\author{
Grand Roman Joldes ${ }^{1}$, Adam Wittek ${ }^{1}$, Mathieu Couton ${ }^{1,3}$, Simon K. Warfield ${ }^{2}$, \\ and Karol Miller ${ }^{1}$ \\ ${ }^{1}$ Intelligent Systems for Medicine Laboratory, The University of Western Australia \\ 35 Stirling Highway, 6009 Crawley/Perth, Western Australia, Australia \\ ${ }^{2}$ Computational Radiology Laboratory, Children's Hospital Boston and \\ Harvard Medical School \\ 300 Longwood Avenue, Boston, MA02115, USA \\ ${ }^{3}$ Institut Francais de Mechanique Avancee IFMA \\ Clermont Ferrand, 63175 Aubiere Cedex, France \\ grandj@mech.uwa.edu.au
}

\begin{abstract}
Patient-specific biomechanical models implemented using specialized nonlinear (i.e. taking into account material and geometric nonlinearities) finite element procedures were applied to predict the deformation field within the brain for five cases of craniotomy-induced brain shift. The procedures utilize the Total Lagrangian formulation with explicit time stepping. The loading was defined by prescribing deformations on the brain surface under the craniotomy. Application of the computed deformation fields to register the preoperative images with the intraoperative ones indicated that the models very accurately predict the intraoperative positions and deformations of the brain anatomical structures for limited information about the brain surface deformations. For each case, it took less than $40 \mathrm{~s}$ to compute the deformation field using a standard personal computer, and less than $4 \mathrm{~s}$ using a Graphics Processing Unit (GPU). The results suggest that nonlinear biomechanical models can be regarded as one possible method of complementing medical image processing techniques when conducting non-rigid registration within the real-time constraints of neurosurgery.
\end{abstract}

\section{Introduction}

Distortion of the preoperative anatomy due to surgical intervention and misalignment between the actual position of pathology and its position determined from the preoperative images are one of the key challenges facing image-guided neurosurgery. A typical example is the craniotomy-induced brain shift that results in movement of the pathology (tumor) and critical healthy tissues. As the contrast and spatial resolution of the intraoperative images are typically inferior to the preoperative ones [1], the highquality preoperative data need to be aligned to the intraoperative brain geometry to retain the preoperative image quality during the surgery. Accurate alignment requires taking into account the brain deformation, which implies nonrigid registration. In recent years image-based methods for nonrigid registration have been improved by 
including the biomechanical models that take into account the mechanical properties of anatomical structures depicted in the image, and, therefore, ensure plausibility of the predicted intraoperative deformation field [2-4]. In most practical cases, such models utilize the finite element method to solve sets of partial differential equations of solid mechanics governing the behavior of continua. In the vast majority of biomechanical models for nonrigid registration linear finite element procedures were used $[2,3,5]$. In these procedures, the brain deformation is assumed to be infinitesimally small, i.e. the equations of solid mechanics are integrated over the initial (undeformed) brain geometry. It has been reported in several studies, that linear finite element procedures can facilitate computations of brain deformation within the real-time constraints of neurosurgery (below $60 \mathrm{~s}$ according to [6]). For instance, Warfield et al. [2] reported the computation time of around $15 \mathrm{~s}$ and Skrinjar et al. [5] - time of $80 \mathrm{~s}$.

However, the brain surface deformations due to craniotomy can exceed $20 \mathrm{~mm}$ [7] which is inconsistent with the infinitesimally small deformation assumption. Therefore, in several studies [4, 8], finite element models utilizing geometrically nonlinear (i.e. finite deformations) formulation of solid mechanics have been used to compute the deformation field within the brain for neuroimage registration. Despite facilitating accurate predictions of the brain deformations, the nonlinear biomechanical models have been, so far, of little practical importance as the algorithms used in such models led to computation times appreciably exceeding the real-time constraints of neurosurgery [9].

Recently, however, specialized nonlinear finite element algorithms and solvers for real-time computation of soft organ deformation for image registration have become available [10-11]. In this study, we use them to compute brain deformation in five cases of craniotomy-induced brain shift. We demonstrate that biomechanical models using specialized nonlinear finite element algorithms facilitate accurate prediction of deformation field within the brain for computation times below $40 \mathrm{~s}$ on a standard personal computer and below $4 \mathrm{~s}$ on a Graphics Processing Unit (GPU).

\section{Methods}

\subsection{Analyzed Cases}

To account for various types of situations that occur in neurosurgery, we analyzed five cases of craniotomy-induced brain shift with tumor (and craniotomy) located anteriorly (cases 1 and 2), laterally (case 3) and posteriorly (cases 4 and 5) (Fig. 1).

\subsection{Construction of Finite Element Meshes for Patient-Specific Brain Models}

Three-dimensional patient-specific brain meshes were constructed from the segmented preoperative magnetic resonance images (MRIs). The segmentation was done using seed growing algorithm followed, in some cases, by manual corrections.

Because of the stringent computation time requirements, the meshes had to be constructed using low order elements that are not computationally expensive. The linear under-integrated hexahedron is the preferred choice. Many algorithms are now available for fast and accurate automatic mesh generation using tetrahedral elements, but not for automatic generation of hexahedral meshes [12]. Template based meshing algorithms could not be used here because of the presence of irregularly placed and 

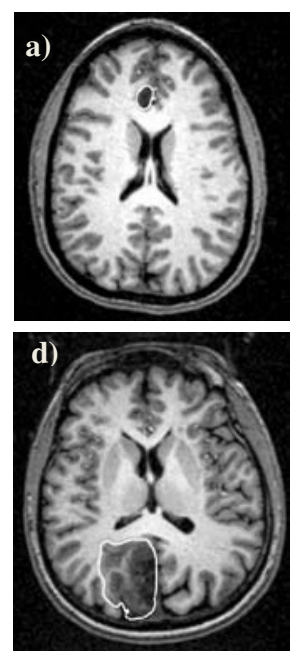
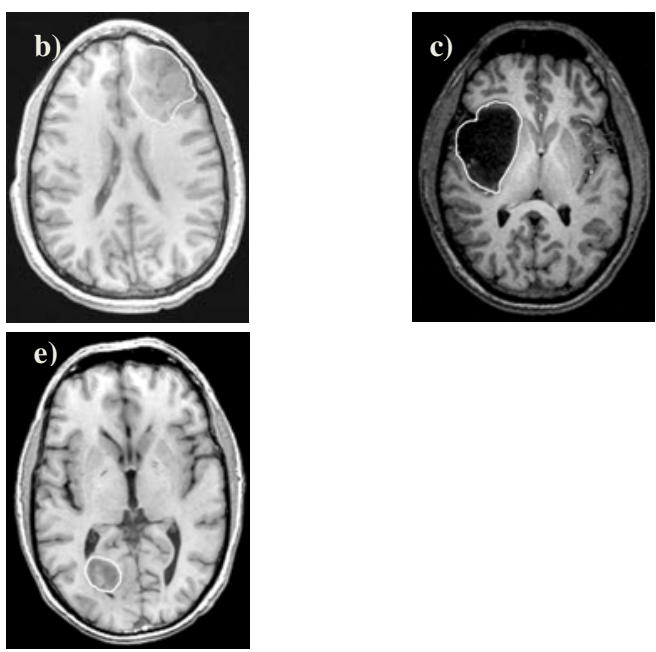

Fig. 1. Preoperative T1 MRIs (inferior view) showing tumor location in the cases analyzed in this study. White lines indicate the tumor segmentations. a) Case 1; b) Case 2; c) Case 3; d) Case 4; and e) Case 5.

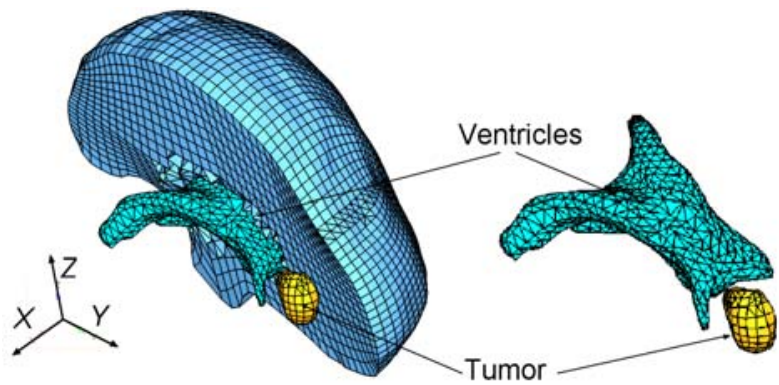

Fig. 2. Typical example (Case 1) of a patient-specific brain mesh built in this study. In this example the ventricles were discretized using tetrahedral elements only.

Table 1. Summary of the patient-specific brain meshes built in this study

\begin{tabular}{lcrrrr}
\hline & Case1 & Case2 & Case3 & Case4 & Case5 \\
\hline Number of Hexahedral Elements & 14447 & 10258 & 10127 & 9032 & 8944 \\
Number of Tetrahedral Elements & 13563 & 20316 & 23275 & 23688 & 21160 \\
Number of Nodes & 18806 & 15433 & 15804 & 14732 & 14069 \\
Number of Degrees of Freedom & 55452 & 45315 & 46896 & 43794 & 42018 \\
\hline
\end{tabular}

shaped tumors. Therefore, to partly automate the meshing, we used mixed meshes consisting of both linear hexahedral and tetrahedral elements (Fig. 2, Table 1). As the brain parenchyma is typically regarded as either incompressible or nearly incompressible $[13,19]$, the linear tetrahedral elements with average nodal pressure ANP 
formulation [14] were used to prevent volumetric locking. The meshes were built using IA-FEMesh (a freely available software toolkit aimed at hexahedral mesh generation developed at the University of Iowa [15]) and HyperMesh (a highperformance commercial finite element mesh generator by Altair of Troy, MI, USA).

\subsection{Biomechanical Model for Brain Shift Computation}

Loading and Boundary Conditions. There are always uncertainties in patientspecific properties of the living tissues. To reduce the effects of such uncertainties, we loaded the models by prescribing displacements on the exposed (due to craniotomy) part of the brain surface. It has been suggested in [9] that for this type of loading, the unknown deformation field within the brain depends very weakly on the mechanical properties. The displacements for loading the models were determined from the segmented preoperative and intraoperative cortical surfaces. The correspondences between the preoperative and intraoperative surfaces were determined by applying the vector-spline regularization algorithm described in [16] to the surface curvature maps.

To define the boundary conditions for the remaining nodes on the brain model surface, a contact interface was defined between the rigid skull model and the part of the brain surface where nodal displacements were not prescribed. Contact formulation described in [17] was used. This formulation prevents the brain surface from penetrating the skull while allowing for frictionless sliding and separation between the brain and skull.

Mechanical Properties for the Models. It has been reported in [9] that, when the geometrical nonlinearity is taken into account, constitutive model of the brain tissue exerts negligible effects on the brain shift prediction. Therefore, we used the simplest hyperelastic model: the neo-Hookean one [18]. Based on the published experimental data [13] a value of $3000 \mathrm{~Pa}$ was used for the parenchyma Young's modulus. The Young's modulus of the tumor was designated a value two times larger than that of the parenchyma, which is consistent with the experimental data of Sinkus et al. [20]. Following [8], we used Poisson's ratio of 0.49 for the brain parenchyma and tumor.

Solution Algorithms. We described the details of the applied algorithms (including their verification and validation) in our previous publications $[10,11,14,21]$. Therefore, only a brief summary is given here. Computational efficiency of the algorithms for integrating the equations of solid mechanics used in this study have been achieved through application of the following two means: 1) Total Lagrangian (TL) formulation [10, 11] for updating the calculated variables; and 2) Explicit Integration in the time domain combined with mass proportional damping. In the Total Lagrangian formulation, all the calculated variables (such as displacements and strains) are referred to the original configuration of the analyzed continuum. The decisive advantage of this formulation is that all derivatives with respect to spatial coordinates can be pre-computed [10]. As indicated in [10], this greatly reduces the computation time in comparison to Updated Lagrangian formulation used in vast majority of commercial finite element solvers (such as e.g. LS-DYNA, ABAQUS).

In explicit time integration, the displacement at time $t+\Delta t$ (where $\Delta t$ is the time step) is solely based on the equilibrium at time $t$. Therefore, no matrix inversion and iterations are required when solving nonlinear problems. In consequence, application 
of explicit integration can reduce the time required to compute the brain deformations by an order of magnitude in comparison to implicit integration typically used in commercial finite element codes for steady state solutions [8].

Accuracy Evaluation. Universally accepted "gold standards" for validation of nonrigid registration techniques have not been developed yet [22]. Objective metrics of the images' alignment can be provided by automated methods using image similarity metrics (such as e.g. Mutual Information and Normalized Cross-Correlation). One of the key deficiencies of such metrics is that they quantify the alignment error in terms that do not have straightforward geometrical (in Euclidean sense) interpretation.

To provide an error measure that enables such interpretation, we compared X, Y and $\mathrm{Z}$ bounds of the ventricles determined from the intraoperative segmentations and obtained by registration (i.e. warping using the predicted deformation field) of the preoperative data. The bounds provide six numbers that can be geometrically interpreted as the $\mathrm{X}, \mathrm{Y}$ and $\mathrm{Z}$ coordinates of vertices $\mathrm{P}_{1}$ and $\mathrm{P}_{2}$ defining a cuboidal box bounding the ventricles (see Fig. 3). The difference between the coordinates of these vertices determined from the intraoperative MRIs and predicted by our biomechanical models was used as a measure of the alignment error. The coordinates of the vertices $\mathrm{P}_{1}$ and $\mathrm{P}_{2}$ can be determined automatically, which makes such difference less prone to subjective errors than the measures based on anatomical landmarks selected by experts. We provide no error measure for the tumor registration as we were not able to reliably quantify the intraoperative bounds of tumors due to limited quality of the intraoperative images.

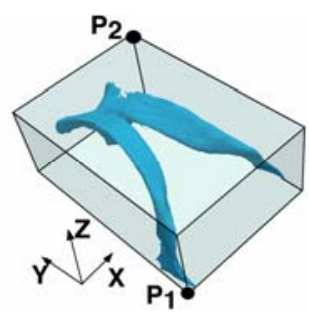

Fig. 3. Definition the ventricles' bounds. Vertices $P 1$ and P2 define a cuboidal box that bounds the ventricles. The box faces are formed by planes perpendicular to $\mathrm{X}, \mathrm{Y}$ and $\mathrm{Z}$ axes.

\section{Results}

The maximum displacement of the cortical surface was $7.7 \mathrm{~mm}$ (Case 2), and the brain maximum (Green) strain predicted by our models was $30 \%$. The computation times on a PC (Intel E6850 dual core $3.00 \mathrm{GHz}$ processor, $4 \mathrm{~GB}$ of internal memory, and Windows XP operating system) varied from $30 \mathrm{~s} \mathrm{(Case} \mathrm{1)} \mathrm{to} 38 \mathrm{~s}$ (Case 5). Following our earlier work [23] on application of Graphics Processing Units (GPUs) for scientific computations, we also implemented our algorithms using the NVIDIA Compute Unified Device Architecture (CUDA). Non-trivial details of this implementation are given in [24]. For the NVIDIA CUDA implementation of our algorithms, the computation times were under $4 \mathrm{~s}$ for all the craniotomy cases analyzed in this 
study. The maximum errors when predicting the intraoperative bounds of the ventricles were $1.6 \mathrm{~mm}$ in $\mathrm{X}$ (lateral) direction, $1.6 \mathrm{~mm}$ in $\mathrm{Y}$ (i.e. anterior-posterior) direction and $2.2 \mathrm{~mm}$ in $\mathrm{Z}$ (inferior-superior) direction (Table 2). These errors compare well with the voxel size $\left(0.86 \times 0.86 \times 2.5 \mathrm{~mm}^{3}\right)$ of the intraoperative images. Detailed comparison of the contours of ventricles in the intraoperative images and the ones predicted by the finite element brain models developed in this study indicate some local misregistration. However, the overall agreement is remarkably good (Fig. 4).

In Table 2, the computation results are presented to one decimal place as it has been reported in the literature [8] that this is approximately the accuracy of finite element computations using the type of finite element algorithms applied in this study.

Table 2. Error in predicting the $\mathrm{X}, \mathrm{Y}$, and $\mathrm{Z}$ coordinates (in millimeters) of vertices $\mathrm{P} 1$ and $\mathrm{P} 2$ defining the bounds of the ventricles in the intraoperative MRIs (see Fig. 3). The directions of the $\mathrm{X}, \mathrm{Y}$, and $\mathrm{Z}$ axes are as in Fig. 3. The numbers in bold font indicate the maximum errors.

\begin{tabular}{c|c|c|c|c|c|c}
\hline & \multicolumn{2}{|c|}{ X Coordinate Error [mm] } & \multicolumn{2}{c|}{ Y Coordinate Error [mm] } & \multicolumn{2}{c}{ Z Coordinate Error [mm] } \\
\cline { 2 - 7 } & P1 & P2 & P1 & P2 & P1 & P2 \\
\hline Case 1 & 0.3 & 0.2 & 0.7 & 1.3 & 0.7 & 0.2 \\
Case 2 & 0.0 & 0.5 & 1.2 & 0.5 & 0.6 & 0.5 \\
Case 3 & $\mathbf{1 . 6}$ & 0.4 & 0.6 & $\mathbf{1 . 6}$ & $\mathbf{2 . 2}$ & 0.1 \\
Case 4 & 0.5 & 0.0 & 0.5 & 0.4 & 0.1 & 0.7 \\
Case 5 & 0.1 & 0.4 & 0.5 & 1.5 & 1.1 & 0.4 \\
\hline
\end{tabular}

a)

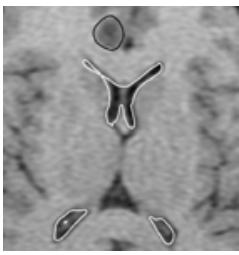

d)

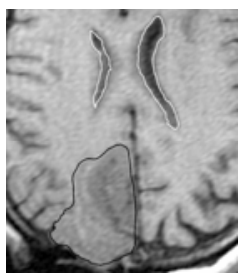

b)

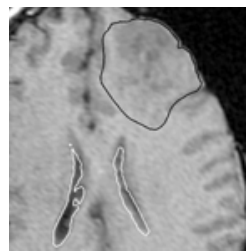

e)

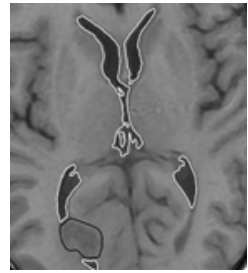

c)

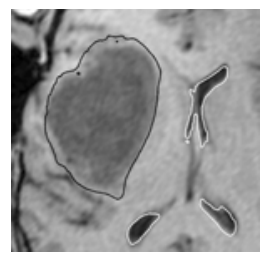

Fig. 4. The registered (i.e. deformed using the calculated deformation field) preoperative contours of ventricles and tumor are imposed on the intraoperative images. The images were cropped and enlarged. a) Case 1; b) Case 2; c) Case 3; d) Case 4; and e) Case 5.

\section{Discussion}

Instead of relying on unrealistic linearization (i.e. assumption about infinitesimally small brain deformation during craniotomy and linear stress-strain relationship of brain tissue) used so far in biomechanical models to satisfy real-time constraints of 
neurosurgery, we applied specialized fully nonlinear (i.e. including both geometrical and material nonlinearities) finite element procedures and predicted deformation field within the brain due to craniotomy-induced brain shift in less than $40 \mathrm{~s}$ using standard personal computer with a single dual-core processor, and less than 4 s using Graphics Processing Unit.

Thus, our results indicate that accurate computations of deformation field within the brain by means of state-of-the-art finite element procedures utilizing fully nonlinear formulation of solid mechanics can be achieved in real time without advanced computer hardware. This is an important step in enabling application of such formulation in neuroimage registration practice. However, before nonlinear computational biomechanical models can become a part of clinical systems for image-guided neurosurgery, reliability and accuracy of such models must be confirmed against much larger data sample than five cases of craniotomy-induced brain shift analyzed in this study.

Acknowledgments. The financial support of the Australian Research Council (Grants DP0664534, DP1092893, DP0770275 and LX0774754), National Institute of Health (Grants R03 CA126466, R01 RR021885, R01 GM074068 and R01 EB008015), CIMIT, and Australian Academy of Science is gratefully acknowledged. We thank Prof. Ron Kikinis of Brigham and Women's Hospital for very helpful suggestions.

\section{References}

1. Warfield, S.K., Haker, S.J., Talos, I.F., Kemper, C.A., Weisenfeld, N., Mewes, A.U.J., Goldberg-Zimring, D., Zou, K.H., Westin, C.F., Wells, W.M., Tempany, C.M.C., Golby, A., Black, P.M., Jolesz, F.A., Kikinis, R.: Capturing Intraoperative Deformations: Research Experience at Brigham and Womens's Hospital. Medical Image Analysis 9, 145-162 (2005)

2. Warfield, S.K., Talos, F., Tei, A., Bharatha, A., Nabavi, A., Ferrant, M., Black, P.M., Jolesz, F.A., Kikinis, R.: Real-Time Registration of Volumetric Brain MRI by Biomechanical Simulation of Deformation During Image Guided Surgery. Computing and Visualization in Science 5, 3-11 (2002)

3. Archip, N., Clatz, O., Whalen, S., Kacher, D., Fedorov, A., Kot, A., Chrisochoides, N., Jolesz, F., Golby, A., Black, P.M., Warfield, S.K.: Non-Rigid Alignment of Pre-Operative MRI, fMRI, and DT-MRI with Intra-Operative MRI for Enhanced Visualization and Navigation in Image-Guided Neurosurgery. NeuroImage 35, 609-624 (2007)

4. Hu, J., Jin, X., Lee, J.B., Zhang, L.Z., Chaudhary, V., Guthikonda, M., Yang, K.H., King, A.I.: Intraoperative Brain Shift Prediction Using a 3D Inhomogeneous Patient-Specific Finite Element Model. Journal of Neurosurgery 106, 164-169 (2007)

5. Skrinjar, O., Nabavi, A., Duncan, J.: Model-Driven Brain Shift Compensation. Medical Image Analysis 6, 361-373 (2002)

6. Gobbi, D.G.: Brain Deformation Correction Using Interactive 3D Ultrasound Imaging. PhD Thesis. Faculty of Medicine, University of Western Ontario, London, Canada (2003)

7. Roberts, D.W., Hartov, A., Kennedy, F.E., Miga, M.I., Paulsen, K.D.: Intraoperative Brain Shift and Deformation: A Quantitative Analysis of Cortical displacement in 28 Cases. Neurosurgery 43, 749-758 (1998)

8. Wittek, A., Miller, K., Kikinis, R., Warfield, S.K.: Patient-Specific Model of Brain Deformation: Application to Medical Image Registration. Journal of Biomechanics 40, 919-929 (2007) 
9. Wittek, A., Hawkins, T., Miller, K.: On the Unimportance of Constitutive Models in Computing Brain Deformation for Image-Guided Surgery. Biomechanics and Modeling in Mechanobiology 8, 77-84 (2009)

10. Miller, K., Joldes, G., Lance, D., Wittek, A.: Total Lagrangian Explicit Dynamics Finite Element Algorithm for Computing Soft Tissue Deformation. Communications in Numerical Methods in Engineering 23, 121-134 (2007)

11. Joldes, G.R., Wittek, A., Miller, K.: Suite of Finite Element Algorithms for Accurate Computation of Soft Tissue Deformation for Surgical Simulation. Medical Image Analysis (in press) (2008), doi:10.1016/j.media.2008.12.001

12. Viceconti, M., Taddei, F.: Automatic Generation of Finite Element Meshes from Computed Tomography Data. Critical Reviews in Biomedical Engineering 31, $27-72$ (2003)

13. Miller, K., Chinzei, K.: Mechanical Properties of Brain Tissue in Tension. Journal of Biomechanics 35, 483-490 (2002)

14. Joldes, G.R., Wittek, A., Miller, K.: Non-locking Tetrahedral Finite Element for Surgical Simulation. Communications in Numerical Methods in Engineering (in press) (2008), doi: $10.1002 / \mathrm{cnm} .1185$

15. Grosland, N.M., Shivanna, K.H., Magnotta, V.A., Kallemeyn, N.A., DeVries, N.A., Tadepalli, S.C., Lisle, C.: IA-FEMesh: An Open-Source, Interactive, Multiblock Approach to Anatomic Finite Element Model Development. Computer Methods and Programs in Biomedicine (in press, 2009), doi: 10.1016/j.cmpb.2008.12.003

16. Arganda-Carreras, I., Sorzano, C.O.S., Marabini, R., Carazo, J.M., Ortiz-de-Solorzano, C., Kybic, J.: Consistent and elastic registration of histological sections using vector-spline regularization. In: Beichel, R.R., Sonka, M. (eds.) CVAMIA 2006. LNCS, vol. 4241, pp. 85-95. Springer, Heidelberg (2006)

17. Joldes, G., Wittek, A., Miller, K., Morriss, L.: Realistic and Efficient Brain-Skull Interaction Model for Brain Shift Computation. In: Computational Biomechanics for Medicine III (MICCAI 2008 Associated Workshop), pp. 95-105 (2008)

18. Yeoh, O.H.: Some Forms of Strain-Energy Function for Rubber. Rubber Chemistry and Technology 66, 754-771 (1993)

19. Miller, K., Chinzei, K.: Constitutive Modeling of Brain Tissue: Experiment and Theory. Journal of Biomechanics 30, 1115-1121 (1997)

20. Sinkus, R., Tanter, M., Xydeas, T., Catheline, S., Bercoff, J., Fink, M.: Viscoelastic Shear Properties of In Vivo Breast Lesions Measured by MR Elastography. Magnetic Resonance Imaging 23, 159-165 (2005)

21. Joldes, G.R., Wittek, A., Miller, K.: An Efficient Hourglass Control Implementation for the Uniform Strain Hexahedron Using the Total Lagrangian Formulation. Communications in Numerical Methods in Engineering 24, 1315-1323 (2008)

22. Chakravarty, M.M., Sadikot, A.F., Germann, J., Bertrand, G., Collins, D.L.: Towards a Validation of Atlas Warping Techniques. Medical Image Analysis 12, 713-726 (2008)

23. Walsh, T.: Hardware Finite Element Procedures. Internal Report. Intelligent Systems for Medicine Laboratory, The University of Western Australia, Crawley, Australia (2004)

24. Joldes, G.R., Wittek, A., Miller, K.: Real-Time Nonlinear Finite Element Computations on GPU Using CUDA - Application to Neurosurgical Simulation. Computer Methods in Applied Mechanics and Engineering (submitted, 2009) 